\title{
Core-Shell Plasmonic Nanomaterials towards: Dual-Mode Imaging Analysis of Glutathione and Enhanced Chemodynamic Therapy
}

\author{
Jin Wang ${ }^{1}$, Ying-Xue Liu ${ }^{1}$, Xiang-Ling Li ${ }^{1,2 *}$, Hong-Yuan Chen ${ }^{1}$, Jing-Juan $\mathrm{Xu}^{1 *}$
}

\footnotetext{
${ }^{1}$ State Key Laboratory of Analytical Chemistry for Life Science, School of Chemistry and Chemical Engineering, Nanjing University, Nanjing 210023, P.R. China.

${ }^{2}$ College of Life Science and Pharmaceutical Engineering, Nanjing Tech University, Nanjing 211816 , P.R. China.

*Corresponding author: Tel/ fax: +86-25-89687924, Email: xlli@ njtech.edu.cn. (X.-L. Li), xujj@nju.edu.cn (J.-J. Xu)
}

\section{Table of contents}

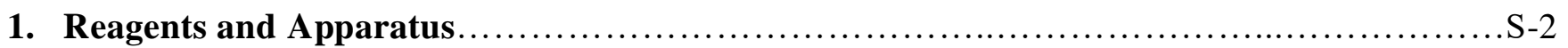

2. Characterization of the Au@MnO2-DNA nanoprobes $\ldots \ldots \ldots \ldots \ldots \ldots \ldots \ldots \ldots \ldots \ldots \ldots \ldots . .3$

3. Quantification of the FAM-ssDNA loaded on each nanoprobe .......................... S-3

4. Traditional FL analysis of GSH by Au@MnO2-DNA nanoprobes............................

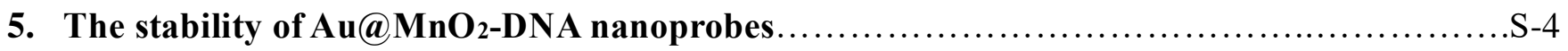

6. Quantitative detection of GSH via Au@ MnO 2 -DNA nanoprobes..........................

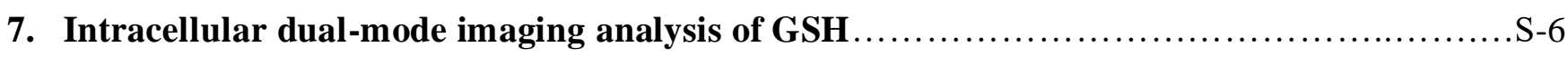

8. Cytotoxicity study and CDT effect of Au@MnO2-DNA nanoprobe........................... 


\section{Reagents and Apparatus}

Reagents. Manganese (II) chloride tetrahydrate (MnCl2 4H2O), 3-(4, 5-dimethyl-2-thiazolyl)-2,5-diphenyl-2Htetrazolium bromide (MTT), hydrogen tetrachloroau-rate (III) hydrate (HAuCl4.3H2O), 2-(N-morpholino) ethanesulfonic acid (MES), 2-ethanesulfonic acid (HEPES), L-glutathione (GSH), N-Ethylmaleimide (NEM), 2',7'-dichlorofluorescein diacetate (DCFH-DA, 97\%), Methylene Blue hydrate and poly (vinylpyrrolidone) (PVP, MW 4000) were purchased from Sigma-Aldrich. KMnO4 was bought from Sinopharm Chemical Reagent Co., $\alpha$-lipoic acid (LPA) was purchased from Aladdin Reagent Co., Ltd, PBS (pH 7.4, 10 mM), high glucose Dulbecco's Modified Eagle Me-dium (DMEM), RPMI-1640 were purchased from KeyGen Biotech. The ssDNA (5'-FAM-TTATAACTATTCCTAAAA-3') used in this work was syn-thesized by Shanghai Sangon Biological Engineering Tech-nology \& Services Co. (Shanghai China).

Apparatus. High-resolution transmission electron microscopy was performed with a JEM-2100 transmission electron microscope (JEOL Ltd., Japan). UV-vis absorption spectra were recorded on a UV-vis spectrophotometer (Nanodrop-2000C, Nanodrop, USA). The $\zeta$-potential was acquired with a Malvern instrument (Nano-Z, Malvern Instruments Ltd., Britain). The traditional FL experiments were conducted on a fluorescence spectrophotometer (F-7000, Hitachi Ltd., Japan). The dark-field measurements were carried out on an inverted microscope (IX71, Olympus) that was equipped with a dark-field condenser $(0.8<\mathrm{NA}<0.92)$ and a $60 \times$ objective lens $(\mathrm{NA}=0.7)$. A $100 \mathrm{~W}$ halogen lamp was used as the white light source. A true-color digital camera (Olympus DP80, Japan) was used to capture the dark-field color images. The scattering light of the nanoparticle was split by a monochromator (Acton SP2358, PI, USA) equipped with a grating (grating density, 300 lines/mm; blazed wavelength, $500 \mathrm{~nm}$ ). The FL images were recorded by an EMCCD (Evolve, Phot ometrics, USA). Confocal fluorescence images of cells were acquired with a TCS SP8 confocal microscopy (Leica, Germany). The cy-tometric analysis was performed on a FC500 Cytometer (Beckman Coulter, United States). 


\section{Characterization of the $\mathrm{Au} @ \mathrm{MnO}_{2}$-DNA nanoprobes}
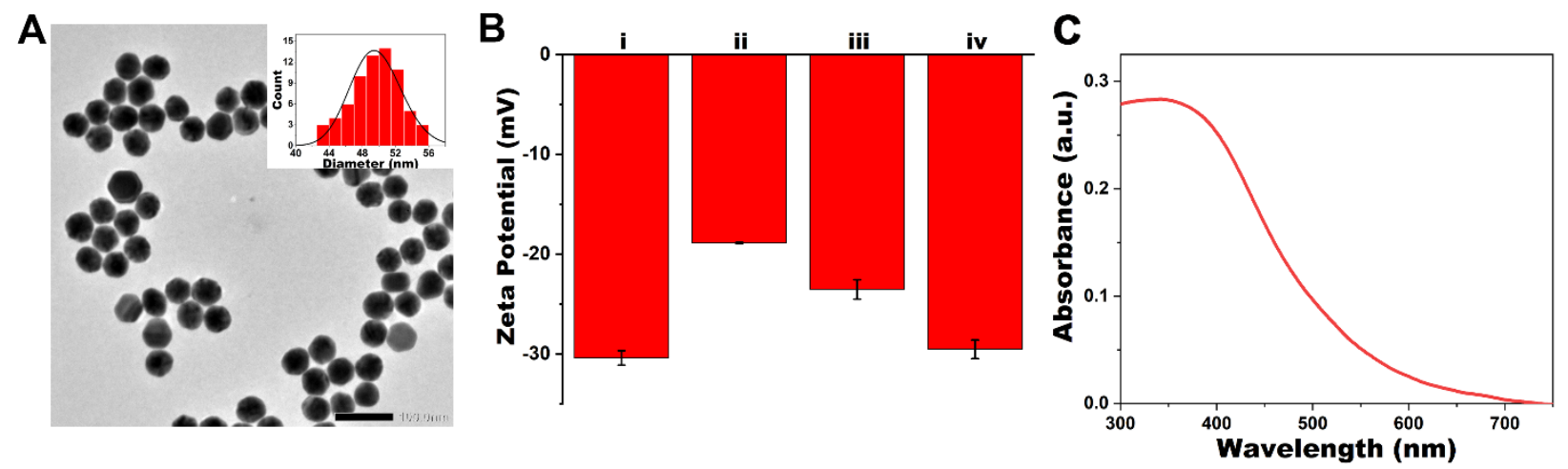

Figure S1 (A) TEM image of the prepared $50 \mathrm{~nm}$ AuNPs. Inset: the particle size distribution of AuNPs. (B) Zeta potential of 50 nm AuNPs (i), AuNPs-PVP (ii), $\mathrm{Au} @ \mathrm{MnO}_{2}$ (iii) and $\mathrm{Au} @ \mathrm{MnO}_{2}-\mathrm{DNA}$ (iv). Error bars indicate the standard deviations of three repetitive experiments. (C) The UV-vis spectrum of $\mathrm{MnO}_{2}$ nanosheet.

\section{Quantification of the FAM-ssDNA loaded on each nanoprobe}

The amount of DNA modified with FAM loaded on each nanoprobe was quantified. First, the final concentration of AuNPs was calculated to be $65.6 \mathrm{pM}$, which is exactly equivalent to the concentration of $\mathrm{Au} @ \mathrm{MnO}_{2}$. Figure $\mathrm{S} 2 \mathrm{~A}$ is the fluorescence spectra of a series of concentrations of ssDNA, and the linear equation is IF $=-1336.64$ $+8459.98 \times \mathrm{C}_{\mathrm{FAM}}(\mu \mathrm{M})$. After incubated $1 \mu \mathrm{M}$ ssDNA with $\mathrm{Au} @ \mathrm{MnO}_{2}$ for $12 \mathrm{~h}$, the fluorescence intensity of the supernatant was $\sim 3824$. Therefore, the concentration of ssDNA in the supernatant was calculated to be 0.61 $\mu \mathrm{M}$ by substituting the above formula. The amount of ssDNA adsorbed on each probe was $\sim 5945$.
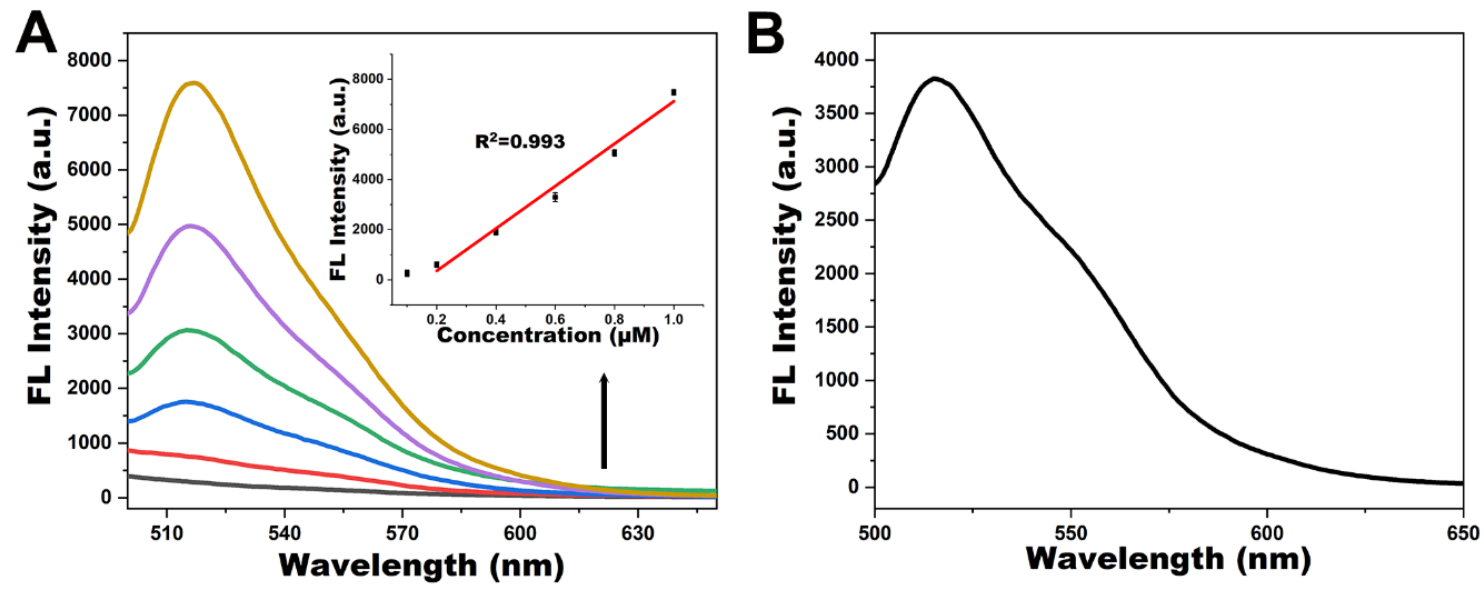

Figure S2 (A) Fluorescence spectra of different concentrations of ssDNA-FAM, the arrow indicated the increasing concentration, inset: the linear relationship between intensity and concentration. (B) Fluorescence emission spectra of FAM in supernatant after the modifying process. $\left(\lambda_{\mathrm{ex}}=492 \mathrm{~nm}, \lambda_{\mathrm{em}}=518 \mathrm{~nm}\right)$. 


\section{Traditional FL analysis of GSH by Au@ MnO2-DNA nanoprobes}
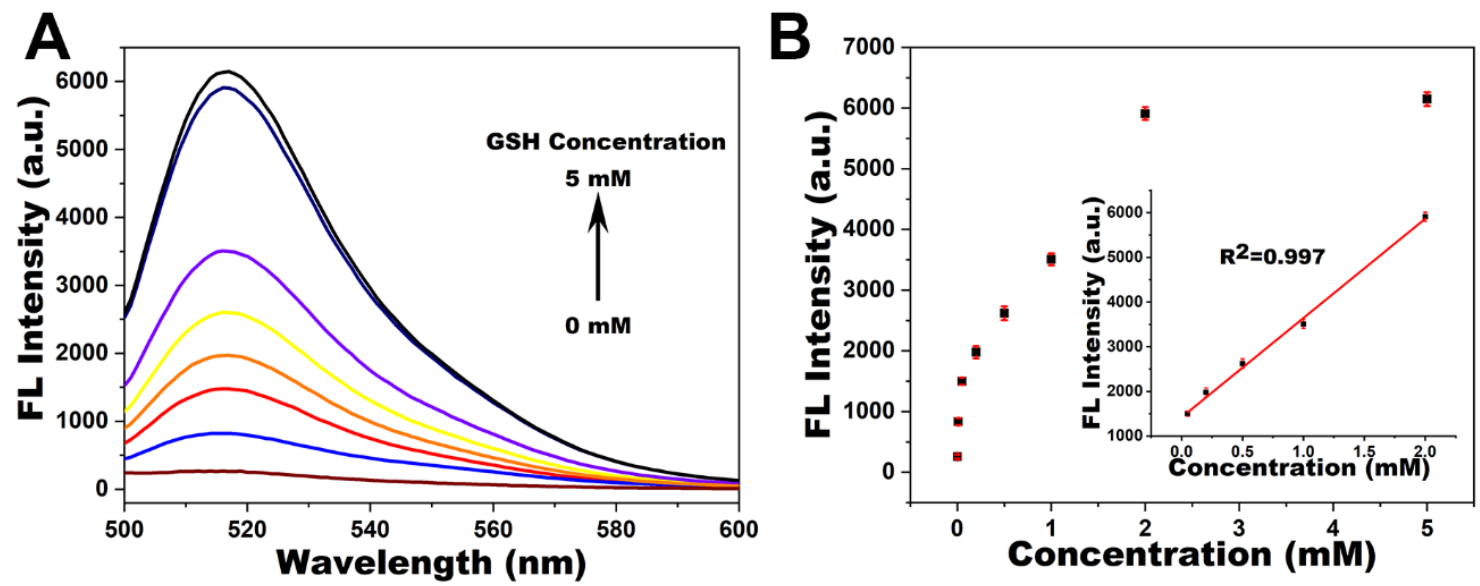

Figure S3 The fluorescence spectral response of $\mathrm{Au} @ \mathrm{MnO}_{2}$-DNA after incubation with GSH of different concentrations $(0-5 \mathrm{mM})$ in phosphate buffer $(\mathrm{pH} 7.0)$. $\left(\lambda_{\mathrm{ex}}=490 \mathrm{~nm}, \lambda_{\mathrm{em}}=520 \mathrm{~nm}\right)$.

\section{The stability of Au@MnO2-DNA nanoprobes}
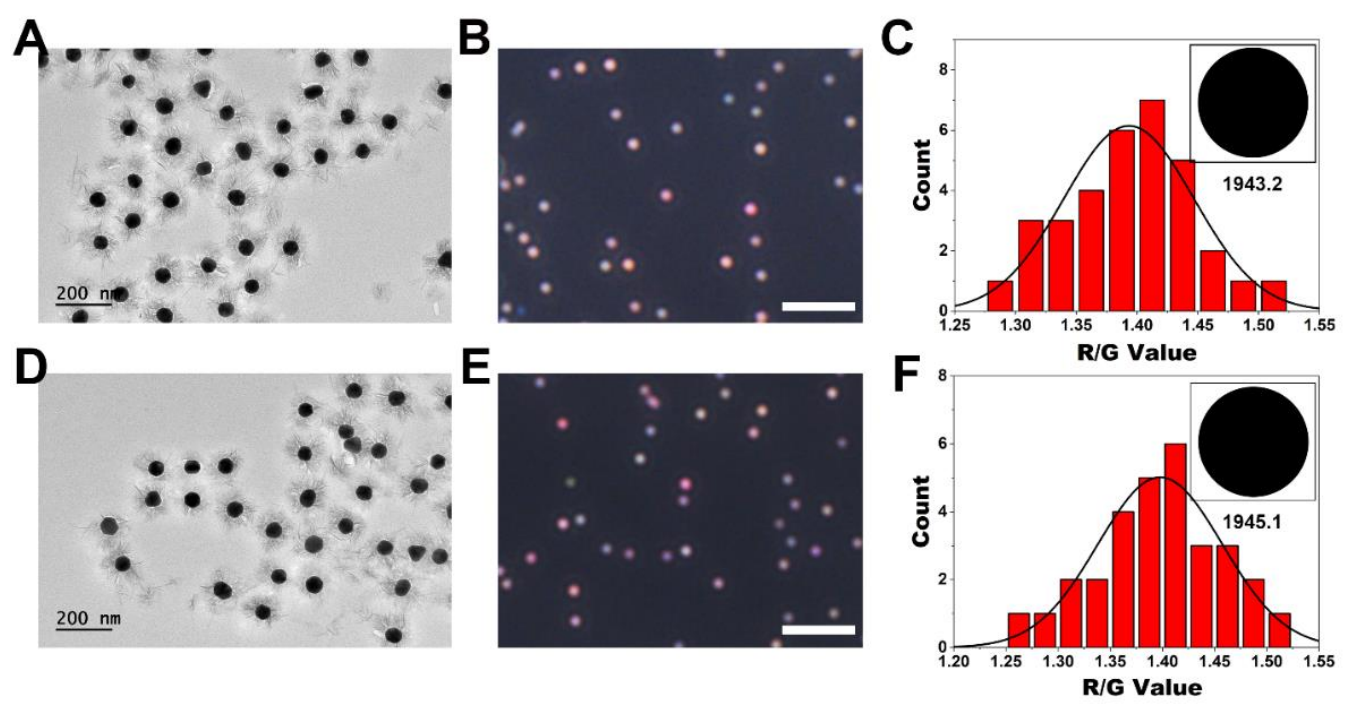

Figure S4 (A)-(C) The stability of $\mathrm{Au} @ \mathrm{MnO}_{2}$-DNA NPs dispersed in PBS for 48 h. (A) TEM images; (B) DFM images; the scar bar is $2 \mu \mathrm{m}$. (C) The $R / G$ value analysis of nanoprobe in (B). Insert: FL image captured by EMCCD. (D)-(E) The stability of $\mathrm{Au} @ \mathrm{MnO}_{2}-\mathrm{DNA} N$ s dispersed in cell culture for 48 h. (D) TEM images; (E) DFM images; the scar bar is $2 \mu \mathrm{m}$ (F) The $\mathrm{R} / \mathrm{G}$ value analysis of nanoprobe in (E). Insert: FL image captured by EMCCD. 

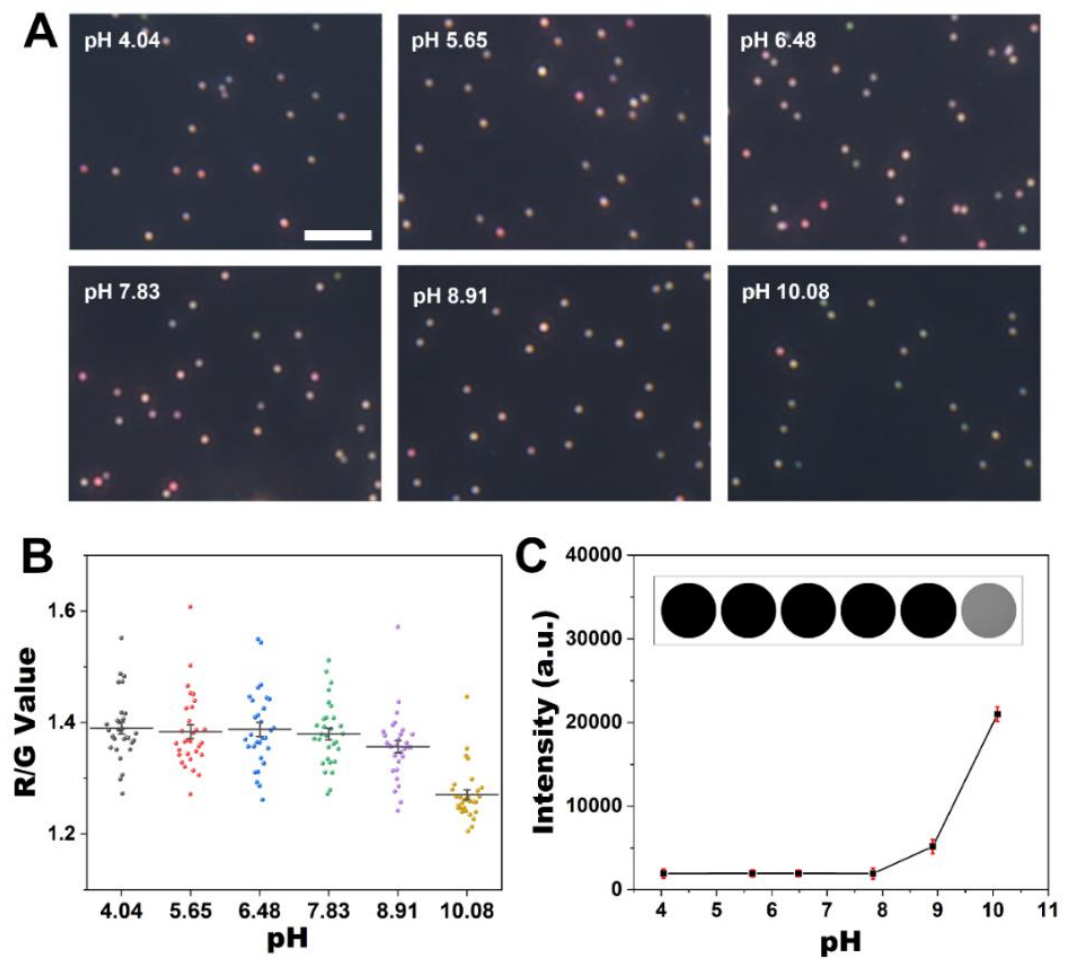

Figure S5 (A) DFM images of $\mathrm{Au} @ \mathrm{MnO}_{2}$-DNA NPs in different pH solutions. The scar bar is $5 \mu \mathrm{m}$. (B) The $\mathrm{R} / \mathrm{G}$ value analysis of nanoprobes in different $\mathrm{pH}$ conditions. (C) The fluorescence intensity under different $\mathrm{pH}$ conditions. Insert: the corresponding FL images.

6. Quantitative detection of GSH via Au@ MnO2-DNA nanoprobes

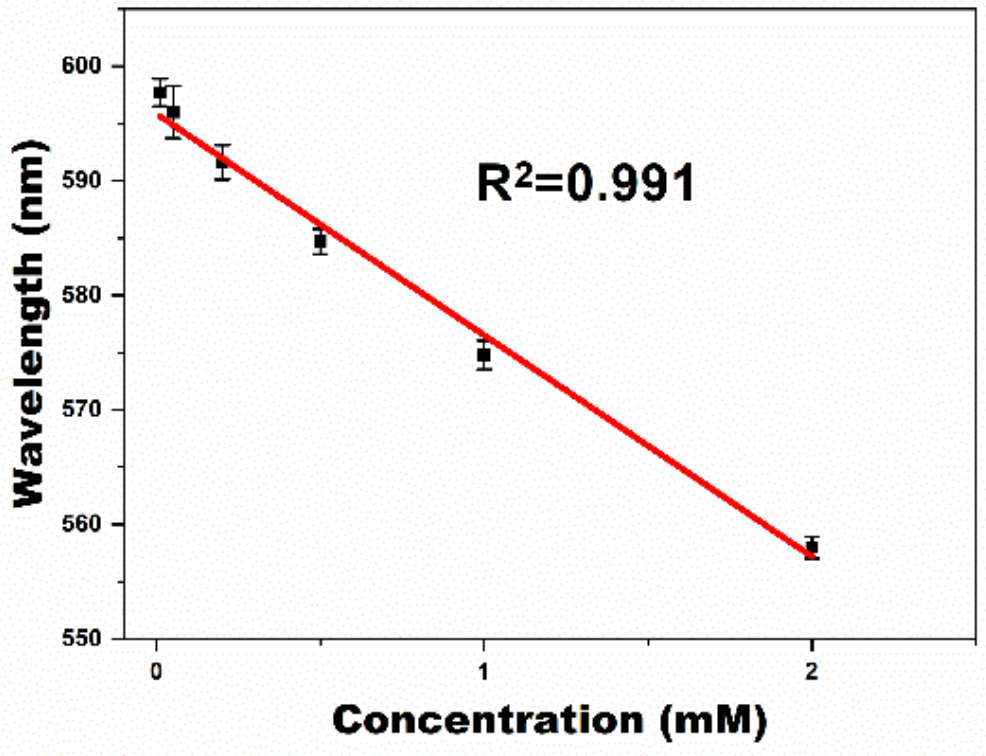

Figure S6 Calibration curve of the wavelengths of scattering peak against GSH concentration. 

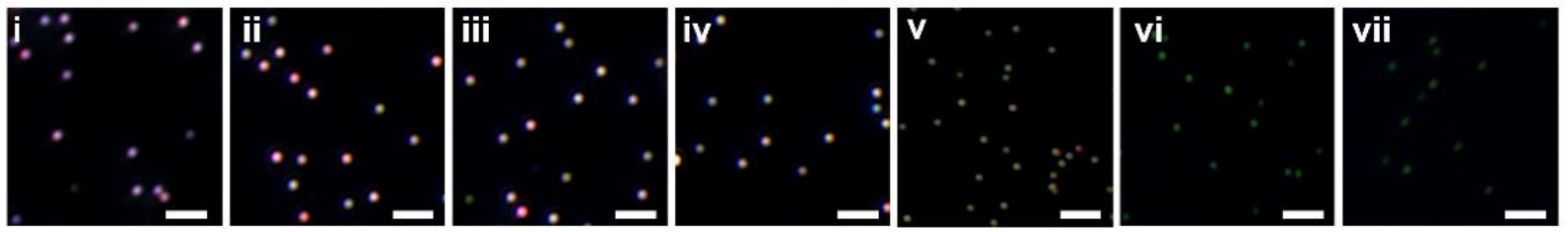

Figure S7 DFM images of $\mathrm{Au} @ \mathrm{MnO}_{2}$-DNA nanoprobes after addition of different concentrations of GSH (ivii: $0.01,0.05,0.2,0.5,1,2$ and $5 \mathrm{mM})$. The scar bar is $2 \mu \mathrm{m}$.

\section{Intracellular dual-mode imaging analysis of GSH}

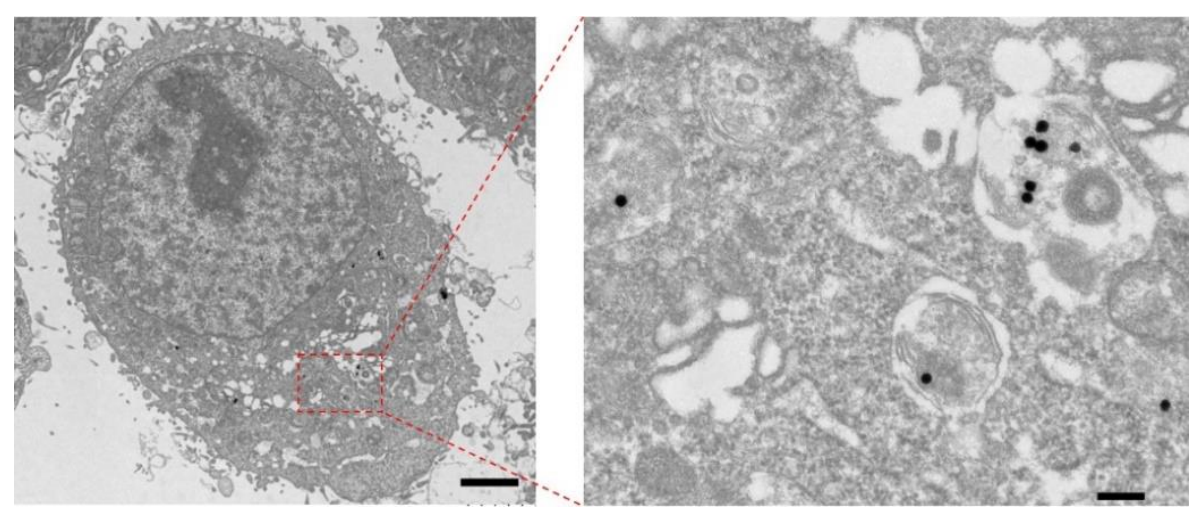

Figure S8 TEM images of Au@ $\mathrm{MnO}_{2}$-DNA NPs incubated with HeLa cells. Scale bar: $2 \mu \mathrm{m}$ (left), $200 \mathrm{~nm}$ (right). 


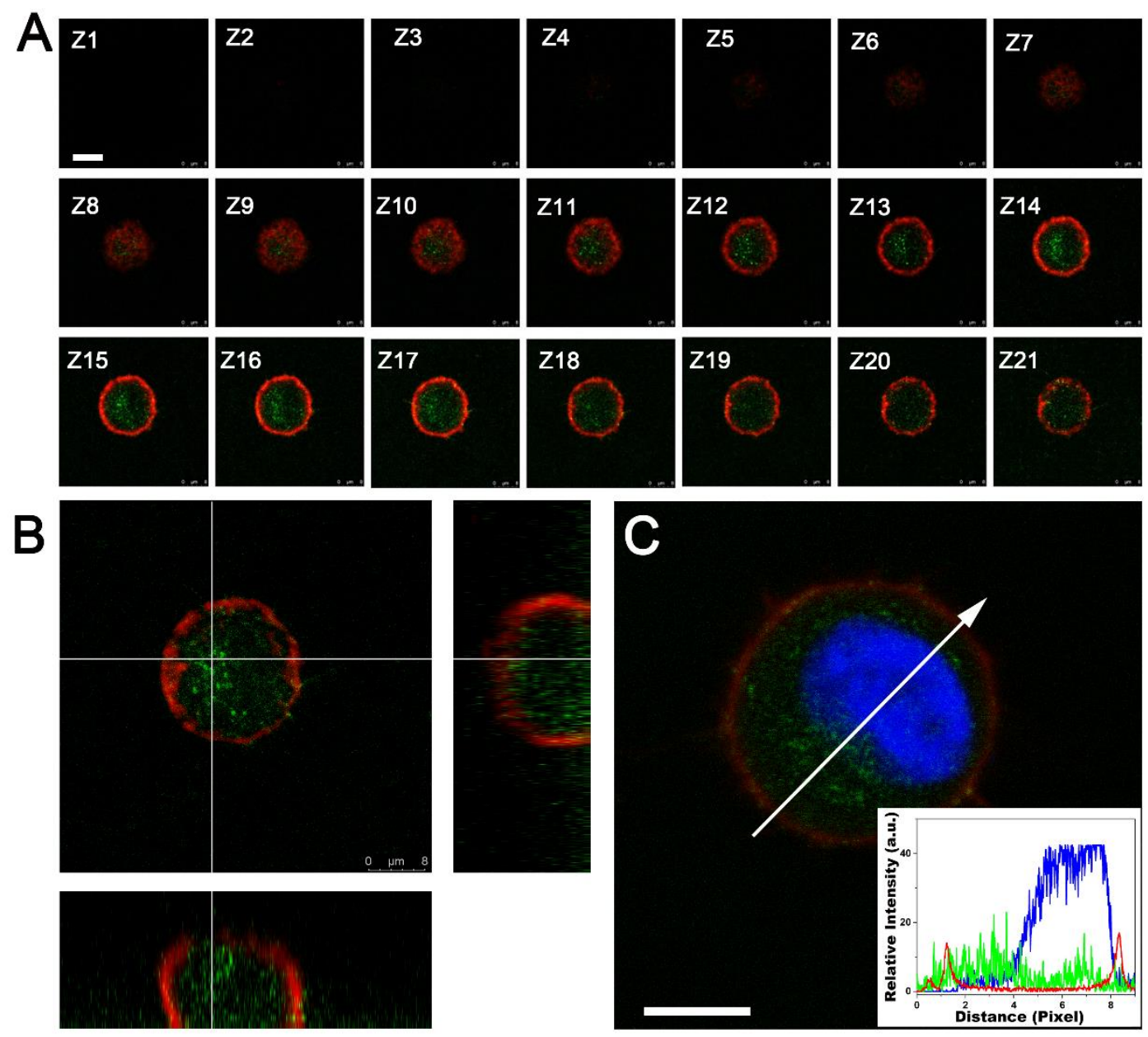

Figure S9 (A) Z-stack images of the merged fluorescence of FAM and CellMask ${ }^{\mathrm{TM}}$ Deep Red Plasma membrane Stain in the HeLa cell after incubated with the probe for $4 \mathrm{~h}$. The images were taken in a series of 21 -step (0.68 $\mu \mathrm{m}$-step-sizes) measurements. (B) The cross-sectional views from $3 \mathrm{D}$ merge images of a $\mathrm{z}$-stack analysis obtained with Leica 3D viewer. (C) Counterstaining analysis of CellMask ${ }^{\mathrm{TM}}$ Deep Red Plasma membrane Stain, $\mathrm{Au} @ \mathrm{MnO}_{2}$-DNA NPs, and Hoechst 33342 in HeLa cells. Inset: fluorescence intensity of CellMask ${ }^{\mathrm{TM}}$ Deep Red Plasma membrane Stain (red line), FAM (green line) and Hoechst 33342 (blue lines) corresponding to the straight line with arrow in $(C)$. Scale bars: $10 \mu \mathrm{m}$. 

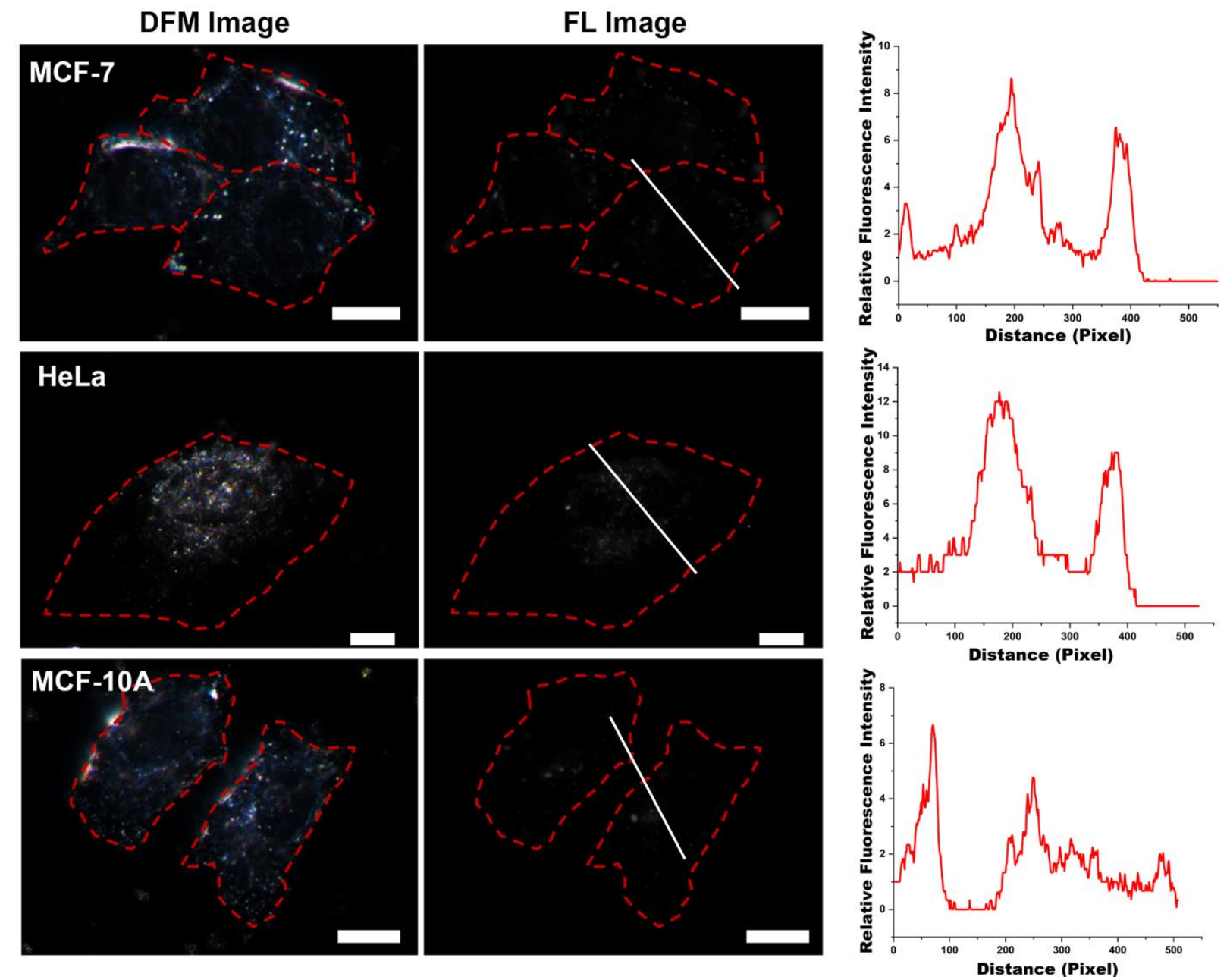

Figure S10 DFM and Fluorescence images of MCF-7, HeLa and MCF-10A cells captured by EMCCD after being treated with $\mathrm{Au} @ \mathrm{MnO}_{2}$-DNA for $4 \mathrm{~h}$ at $37{ }^{\circ} \mathrm{C}$. Curves at the right were the intensity analysis of the corresponding marked positions (white line in the middle channel). The scar bar is $10 \mu \mathrm{m}$.
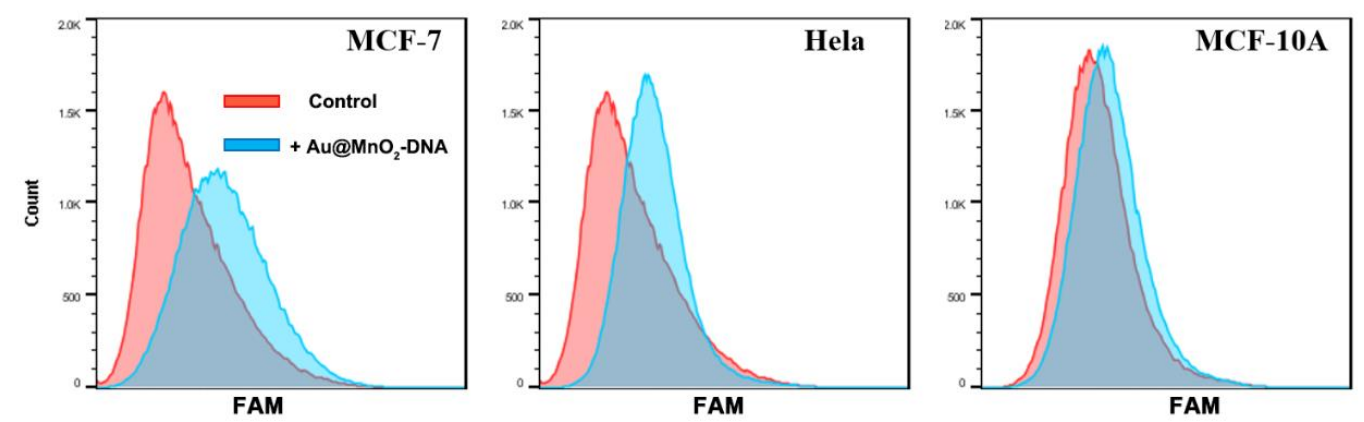

Figure S11 Flow cytometric analysis of MCF-7, HeLa and MCF-10A cells after incubation with $\mathrm{Au} @ \mathrm{MnO}_{2}$-DNA for $4 \mathrm{~h}$ at $37^{\circ} \mathrm{C}$. 


\section{Cytotoxicity study and CDT effect of Au@MnO2-DNA nanoprobe}

$\mathrm{Mn}^{2+}$ from the reaction between $\mathrm{GSH}$ and $\mathrm{MnO}_{2}$ can react with $\mathrm{H}_{2} \mathrm{O}_{2}$ to generate the hydroxyl radicals $(\cdot \mathrm{OH})$ and $\mathrm{Mn}^{3+}$ via the Fenton-like reaction (1 and 2):

$$
\begin{gathered}
\mathrm{MnO}_{2}+2 \mathrm{GSH}+2 \mathrm{H}^{+} \rightarrow \mathrm{Mn}^{2+}+\mathrm{GSSH}+2 \mathrm{H}_{2} \mathrm{O} \text { (1) } \\
\mathrm{Mn}^{2+}+\mathrm{H}_{2} \mathrm{O}_{2} \rightarrow \mathrm{Mn}^{3+}+\cdot \mathrm{OH}+\mathrm{OH}^{-}
\end{gathered}
$$

The following Figure S11 and Figure S12 are the verification of the product $\bullet \mathrm{OH}$.
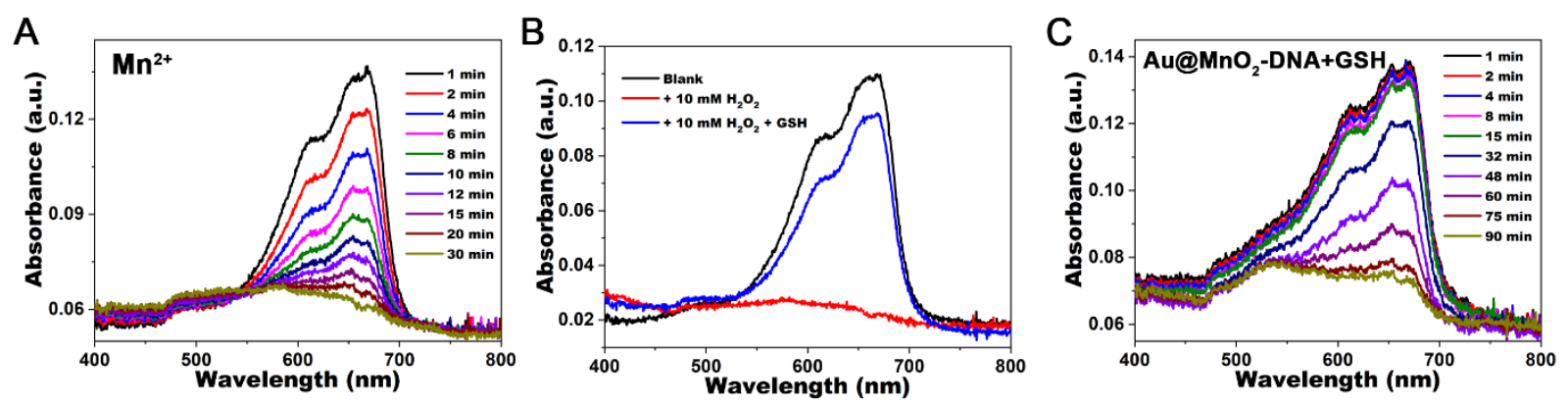

Figure S12 (A) Time-dependence MB degradation in the presence of $\mathrm{Mn}^{2+}$. (B) MB degradation by $\mathrm{Mn}^{2+}$ mediated Fenton-like reaction in the presence or absence of GSH. (C) Time-dependence MB degradation in the presence of $\mathrm{Au} @ \mathrm{MnO}_{2}$-DNA and GSH.

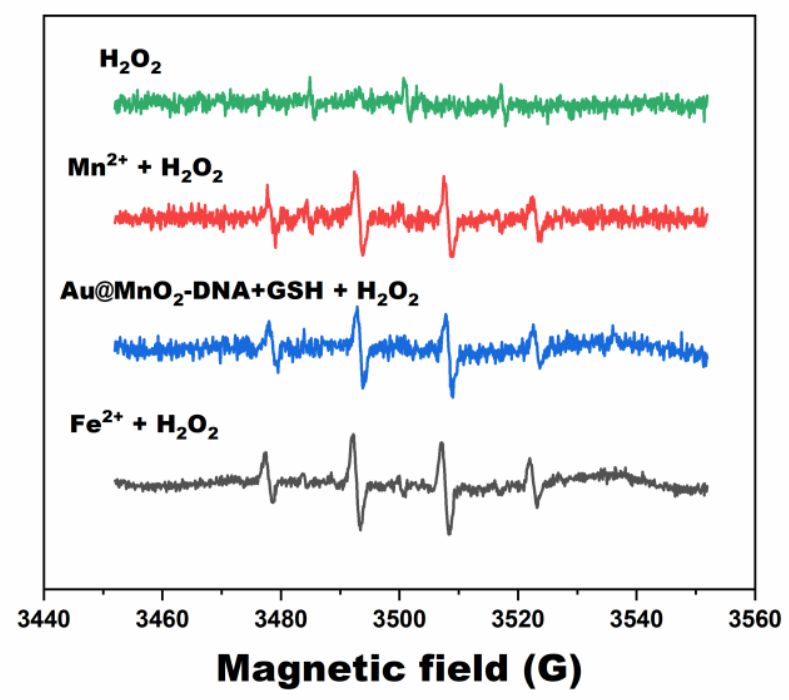

Figure S13 Electron spin resonance (ESR) measurement was applied to detect the generation of $\bullet \mathrm{OH}$ using DMPO. After mixing $\mathrm{H}_{2} \mathrm{O}_{2}(200 \mu \mathrm{M})$ and DMPO; $\mathrm{H}_{2} \mathrm{O}_{2}(200 \mu \mathrm{M})$, DMPO and $\mathrm{Mn}^{2+} ; \mathrm{H}_{2} \mathrm{O}_{2}(200 \mu \mathrm{M})$, DMPO, and the mixture of $\mathrm{Au} @ \mathrm{MnO}_{2}$-DNA NPs and GSH; $\mathrm{H}_{2} \mathrm{O}_{2}(200 \mu \mathrm{M})$, DMPO and $\mathrm{Fe}^{2+}$, the ESR spectrum was measured. The ESR spectra of $\mathrm{H}_{2} \mathrm{O}_{2}$ in the presence of $\mathrm{Mn}^{2+}$ clearly showed a characteristic quartet signal (1:2:2:1) of DMPO-OH, just as the $\mathrm{Fe}^{2+}$, indicating the generation of $\bullet \mathrm{OH}$ species via the Fenton-like reaction mediated catalysis. Similarly, the characteristic ESR spectrum of $\bullet \mathrm{OH}$ appeared when $\mathrm{H}_{2} \mathrm{O}_{2}$ reacted with a 
mixture of $\mathrm{Au} @ \mathrm{MnO}_{2}$-DNA and GSH, further confirming that the nanoprobe could react with GSH to produce $\mathrm{Mn}^{2+}$, which further catalyzed the generation of $\bullet \mathrm{OH}$ from $\mathrm{H}_{2} \mathrm{O}_{2}$.

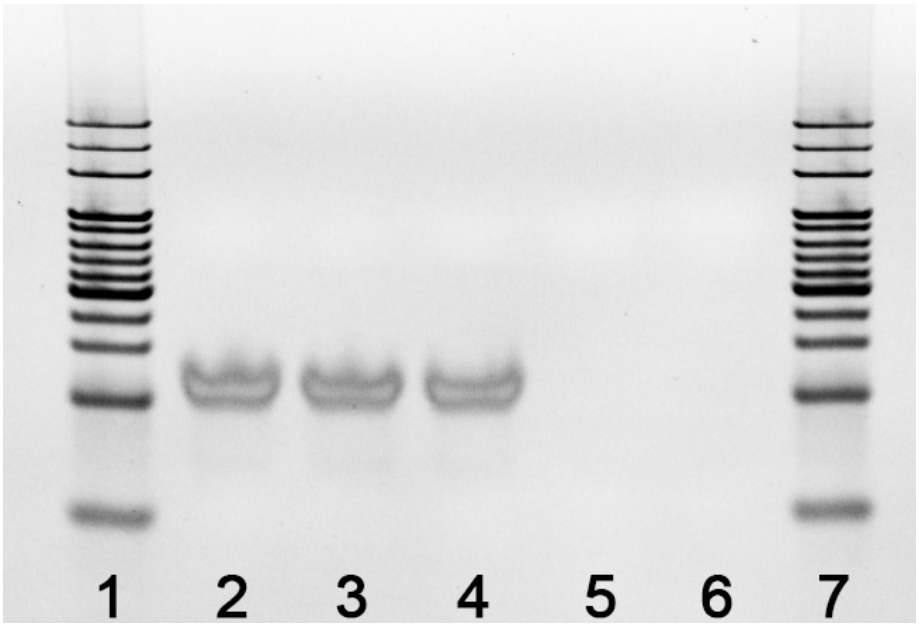

Figure S14 Polyacrylamide gel electrophoresis confirmed $\bullet \mathrm{OH}$ damage to DNA. Lane 1 and lane 7: DNA ladder; lane 2: ssDNA; lane 3: ssDNA $+\mathrm{Mn}^{2+}$; lane 4: ssDNA $+\mathrm{H}_{2} \mathrm{O}_{2}$; lane 5: ssDNA + $\mathrm{Mn}^{2+}+\mathrm{H}_{2} \mathrm{O}_{2}$; lane 6: ssDNA + $\mathrm{Au} @ \mathrm{MnO}_{2}-\mathrm{DNA}+\mathrm{GSH}+\mathrm{H}_{2} \mathrm{O}_{2}$. The results revealed that the ssDNA of 67-bases was broken, which confirmed that the generated $\cdot \mathrm{OH}$ would damage the genetic material and then induce cell apoptosis. (ssDNA: a random single-stranded DNA of 67-bases, GAC ACT GAG AGA TGA AGA TGA AGC CAT ACC GCT TCA TCT TCA TCT CTC TAG GCA CCC ATG TAC AGT C).
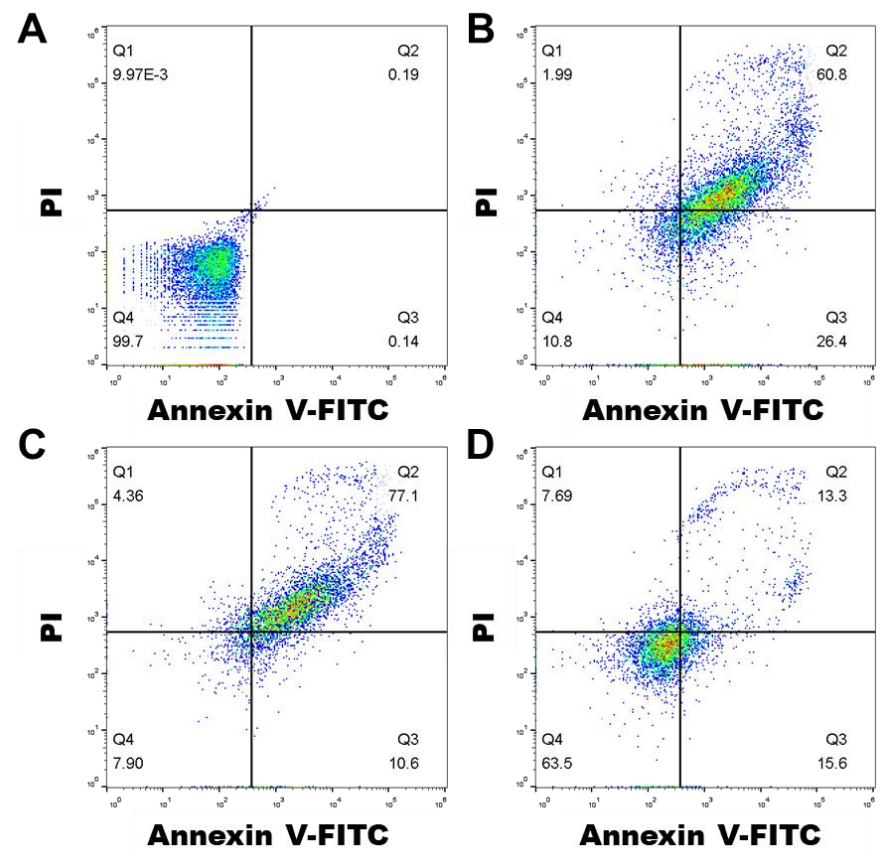

Figure S15 Flow cytometry of HeLa cells treated with (A) control, (B) $\mathrm{Au} @ \mathrm{MnO}_{2}-\mathrm{DNA} N \mathrm{NP}$, (C) LPA and $\mathrm{Au} @ \mathrm{MnO}_{2}$-DNA NPs, (D) NEM and $\mathrm{Au} @ \mathrm{MnO}_{2}$-DNA NPs for $24 \mathrm{~h}$ and stained with Annexin V-FITC/PI apoptosis kit. Q1, necrotic cells; Q2, late apoptotic cells; Q3, early apoptotic cells; Q4, living cells. Inserted numbers in the profiles indicate the percentage of the cells present in this area. 